\title{
Effect of Gastrointestinal Events on Treatment Patterns, Discontinuation, Resource Utilization, and Cost in Osteoporosis: An Analysis Using Danish Health Registries
}

\begin{abstract}
Conclusions: In Danish women aged $\geq 55$, GI events decreased the likelihood of OP treatment, but not the choice of treatment. In OP patients, GI events increase the odds of discontinuation, and GI events post-OP treatment initiation are associated with more resource use and higher cost.
\end{abstract}

Jakob Kjellberg, ${ }^{A}$ Andreas D. Jorgensen, ${ }^{B}$ Peter Vestergaard, ${ }^{C}$ Rikke Ibsen, ${ }^{D}$ and Arun Krishna

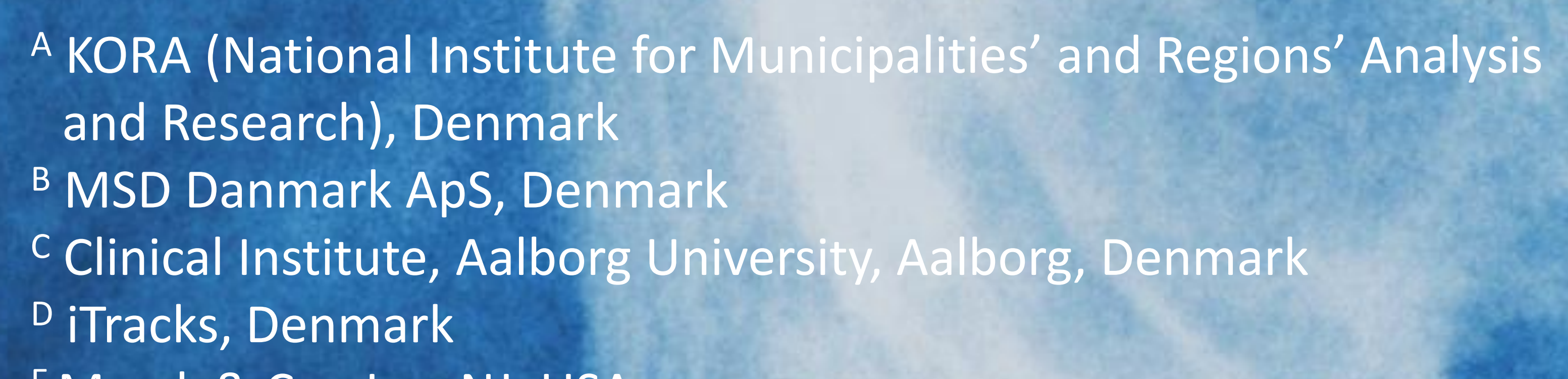

E Merck \& Co., Inc, NJ, USA

\section{Objective}

To investigate the burden and impact of gastrointestinal (GI) events in osteoporosis (OP) patients by (1) examining the association of Gl events with the likelihood of OP treatment initiation; (2) measuring the effect of Gl events on treatment discontinuation; and (3) determining the impact of GI events post-OP treatment on health care resource utilization and cost.

\section{Material and Methods}

All subjects were identified utilizing national health registries which cover the whole Danish population. In women aged $\geq 55$ with an OP diagnosis, we assessed the effect of postdiagnosis $\mathrm{Gl}$ events on treatment initiation $(n=34,614)$. In women aged $\geq 55$ who initiated an oral bisphosphonate, we determined the likelihood of discontinuation after 12 months in patients with and without Gl events ( $n=60,291)$. In a matched subset of post-OP treated patients with $(n=1,373)$ and without ( $n=43,680$ ) $\mathrm{Gl}$ events (cases and controls, respectively), health care resource use and cost were tallied during the year after the first GI event. Subjects in the three analyses were identified within years 2002-2009.

\section{Results}

In objective 1, treatment patterns differed between patients with and without post-diagnosis GI events as $\mathbf{7 1 . 9 \%}$ and 49.4\%, respectively, did not initiate treatment (Figure 1). Hence patients with post-diagnosis GI events had significantly lower odds of treatment initiation (OR $0.43,95 \% \mathrm{Cl} 0.39$ 0.47). Post-diagnosis GI events had no significant effects on the choice of treatment (OR $0.89,95 \% \mathrm{Cl} 0.68-1.16$ ).
Figure 1. Osteoporosis treatment in patients with and without post-diagnosis Gl events

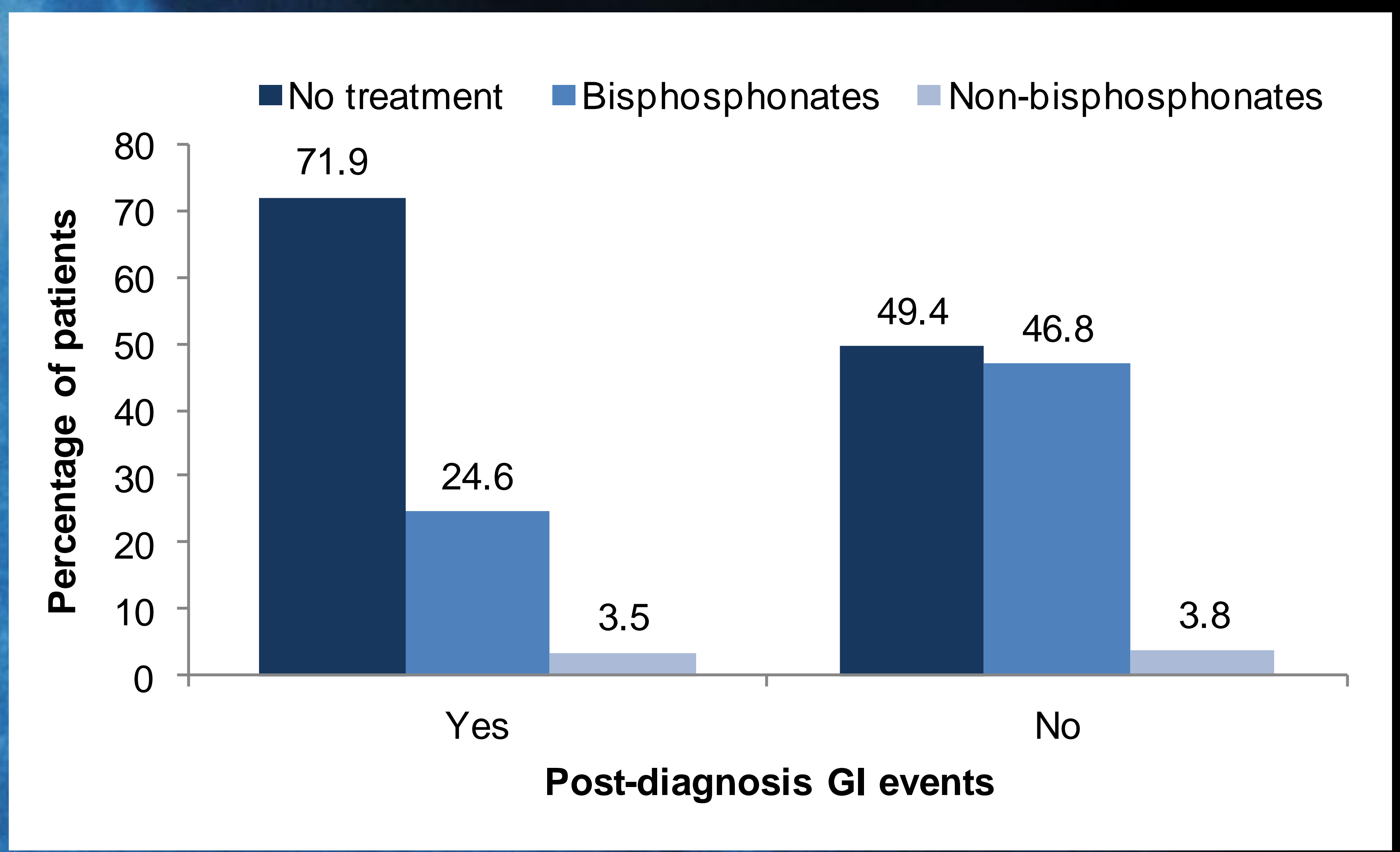

Figure 2A. Impact of post-treatment Gl events on resource use

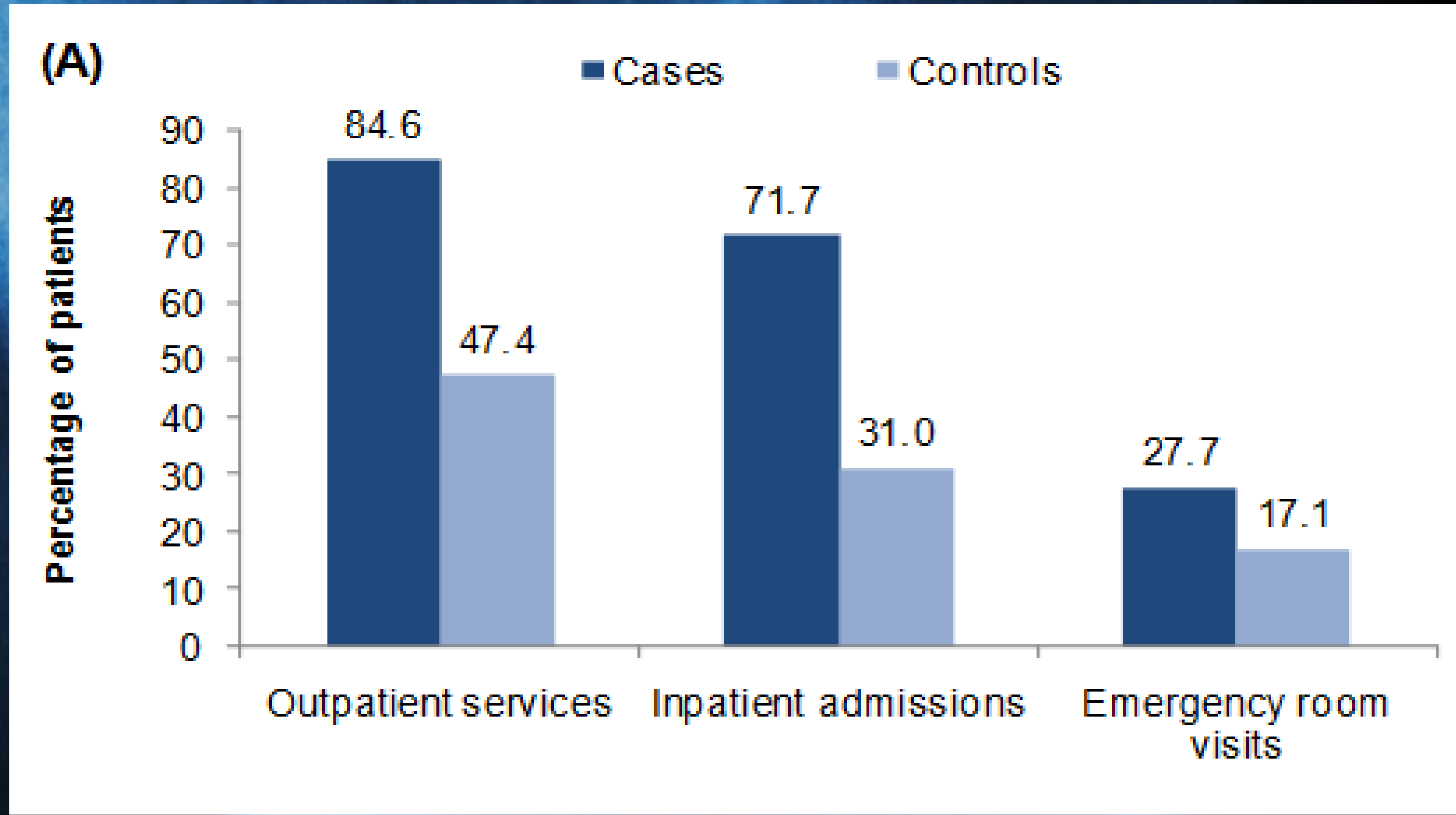

Figure 2B. Impact of post-treatment GI events on health care cost

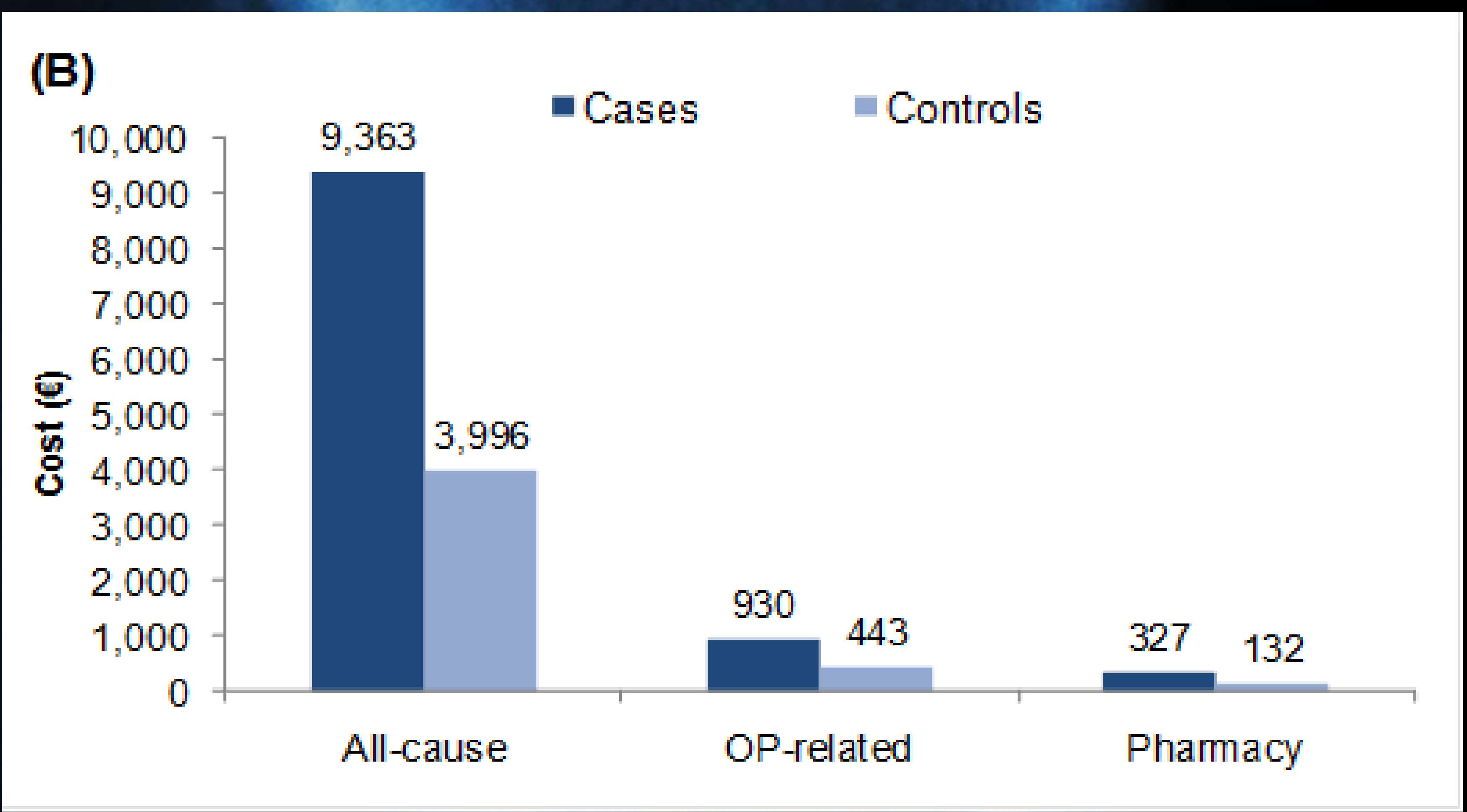

Treatment discontinuation was $36 \%$ more likely at 12 months in patients with post-initiation $\mathrm{Gl}$ events (OR $1.36,95 \% \mathrm{Cl}$ 1.29-1.43).

In the follow-up year, resource use was significantly more frequent in cases (with post-treatment GI events) than controls (Figure 2A) and all-cause cost (mean) accrued by cases $(€ 9,363)$ was more than double that of controls (€3,996; $P<0.001$ ) (Figure 2B). 